\title{
Exosomal microRNA-210 is a potentially non-invasive biomarker for the diagnosis and prognosis of glioma
}

\author{
FENGMING LAN ${ }^{1}$, XIAO YUE ${ }^{2}$ and TINGYI XIA ${ }^{1}$ \\ ${ }^{1}$ Department of Radiotherapy, Chinese PLA General Hospital, Medical School of Chinese PLA, Beijing 100853; \\ ${ }^{2}$ Department of Neurosurgery, The Affiliated Hospital of Xiangnan University, Chenzhou, Hunan 423000, P.R. China
}

Received October 6, 2018; Accepted July 15, 2019

DOI: $10.3892 / \mathrm{ol} .2020 .11249$

\begin{abstract}
MicroRNAs (miRs) transferred by exosomes can function as non-invasive potential biomarkers for the diagnosis and prognosis in various types of cancer. The present study examined the diagnostic and prognostic value of serum exosomal-(exo-)miR-210 levels in association with hypoxic conditions in patients with glioma. Serum levels of exo-miR-210 were determined by quantitative PCR in samples obtained from patients with glioma. Patients were divided into low-and high-expression exo-miR-210 groups according to the median expression value. Statistical analyses were conducted to examine the potential value of exo-miR-210 in predicting the diagnosis and prognosis of patients with glioma. A significant increase in serum exo-miR-210 levels was observed in patients with glioma compared with healthy controls. Additionally, the expression levels of exo-miR-210 were increased with ascending pathological grades. Furthermore, expression levels of miR-210 in serum exosomes from patients with glioblastoma were markedly decreased following surgery and upregulated once more at the recurrences of primary tumors, indicating that exo-miR-210 could reflect alterations in malignant glioma loads. In addition, Kaplan-Meier analysis was performed to analyze overall survival (OS) time. Patients with malignant glioma with high exo-miR-210 expression exhibited a poorer OS compared with patients with low expression. Importantly, univariate and multivariate Cox regression analysis revealed that the expression levels of exo-miR-210 in glioma serum samples were independently associated with OS. Finally, increased serum exo-miR-210 expression was positively associated with high levels of hypoxia-inducible factor 1a and reflected hypoxia in patients with glioma. In conclusion, serum levels of exo-miR-210 may serve as a diagnostic, prognostic and hypoxic biomarker to reflect glioma status and hypoxic signatures.
\end{abstract}

Correspondence to: Professor Tingyi Xia, Department of Radiotherapy, Chinese PLA General Hospital, Medical School of Chinese PLA, 28 Fuxing Road, Haidian, Beijing 100853, P.R. China E-mail: xiatingyi1959@163.com

Key words: glioma, exosome, biomarker, microRNA-210, hypoxia

\section{Introduction}

Glioma is the most common type of primary brain tumor; however, the diagnosis and treatment of glioma remain the most challenging in adults and children (1). Primary glioma is divided into four grades according to the histologic criteria of the World Health Organization (WHO) (2); low-grade (I and II) glioma tumors have a relatively good prognosis, and high-grade tumors (III and IV) are associated with poor clinical outcomes and tumor recurrence (2). In glioblastoma (GBM), the most common and malignant type of glioma, the 5-year survival rate from first diagnosis remains at $<5 \%$, despite progress in multidisciplinary and comprehensive treatment (3). At present, tumor hypoxia has been identified as the primary cause of poor prognosis in patients with GBM (4). Hypoxia is the most important characteristic of aggressive tumors, since the tissues or organs receiving inadequate oxygen, which can lead to rapid tumor growth; the hypoxic tumor microenvironment is involved in different tumor biology processes, including initiation, metabolism, development and metastasis. A previous study has suggested that hypoxic conditions lead to abnormal activation of hypoxia-inducible factors (HIFs), which serve an important role in the regulation of tumor signaling (5). HIF-1 $\alpha$, one of two subunits composing HIF-1, is considered a necessary oxygen-sensitive protein in the regulation of hypoxic signaling pathways (6). The degradation pathway of HIF-1 $\alpha$ is dependent on oxygen; thus, hypoxia in tumor tissues leads to the elevation of HIF-1 $\alpha$ levels (7). It has been reported that HIF- $1 \alpha$ is positively enhanced in most malignant types of cancer and serves an essential role in mediating the hypoxic effects by regulating numerous target genes, including microRNAs (miRNAs/miRs), to adjust the adaptation of tumor cells to hypoxic conditions (8).

miRNAs are a series of evolutionarily conserved small non-coding RNAs that can cause mRNA destabilization and/or translational inhibition by base-pairing with the 3'-untranslated regions of encoding mRNAs (9). A range of miRNAs has been reported as tumor suppressors or oncogenes for the irreplaceable mechanism in tumor initiation and progression, and as therapeutic targets $(10,11)$. Increasing evidence supports the idea that aberrant miRNA expression serves important roles in tumor hypoxia and is associated with HIF-1 $\alpha(12,13)$. HIF-1 $\alpha$ is involved in multidrug resistance by regulating miR-27 in gastric cancer cells (14). Overexpression 
of miR-210 induced by hypoxia is associated with tumor progression and prognosis in upper tract urothelial carcinoma (15). miR-224, which is regulated by hypoxia, promotes the growth, migration and invasion of gastric cancer cells (16). HIF-1 $\alpha$-inducible miR-421 increases metastasis, suppresses apoptosis and results in cisplatin resistance in gastric cancer by targeting E-cadherin and caspase-3 (17).

One of the main HIF-1-dependent genes is carbonic anhydrase 9 (CA9). CA9 is overexpressed in a number of tumor types and serves as a prognostic factor for hypoxic, aggressive and malignant types of cancer (18). CA9 is a transmembrane glycoprotein with enzymatic activity that is located on the cell surface and is inducible by hypoxia (19). Therefore, CA9 has been identified as a potential target for cancer treatment, as it is enriched in hypoxic niches of neoplastic tissues (12).

Tumor hypoxia causes a number of biological alterations in tumor viability, including abnormal secretion and release of exosomes into the tumor microenvironment (20). Exosomes are 30-140 nm nanovesicles carrying genetic materials secreted from various cell types, including cancer cells, released into the tumor microenvironment and maybe taken in by other types of cells (21). miRNAs packaged by exosomes are stable and specific, and thus, appropriate as potential biomarkers to diagnose and distinguish diseases, including various malignancies $(22,23)$. The present study aimed to investigate exosomal-(exo-) miR-210 levels in the serum, which may reflect hypoxic conditions, as a potential diagnostic and prognostic biomarker in patients with glioma.

\section{Materials and methods}

Patients and clinical samples. The present study was approved by the Medical School of Chinese PLA (Beijing, China) and Tianjin Huanhu Hospital (Tianjin, China). In total, 91 patients diagnosed with glioma by clinicopathology and 50 healthy volunteers were recruited from the Medical School of Chinese PLA and Tianjin Huanhu Hospital between October 2011 and July 2014. The study included 52 male and 39 female patients. The median age of the patients was 45 years (range, 19-72 years). The Karnofsky Performance Status (KPS) (24) scores ranged between 70 and 100, and the median score was 90 (Table I). The inclusion criteria were: i) Histological diagnosis of glioma; ii) age between 18 and 80 years; and iii) glioma presented on MRI or PET/CT scans. The exclusion criteria were: i) Severe systemic infection and organ failure; ii) pregnancy; iii) severe immunological disorders (autoimmune disease, immunosuppression); iv) multiple cancers; and v) anaphylaxis induced by synthetic peptides. None of the patients were treated with chemotherapy or radiotherapy prior to surgery. All procedures in the present study involving human participants were performed in accordance with relevant ethical standards. Blood samples were collected at the specific time points; pre-operation, post-operation and recurrence, to examine the expression levels of exo-miR-210. Patients with co-morbidities receiving other medication were excluded from the present study. All participants provided written informed consent prior to recruitment. All patients with glioma were followed up at regular intervals of 3 months for up to 5 years. Although the terminal point of the $\mathrm{x}$-axis is 70 months, the survival curve was limited to 60 months. Survival times were calculated from the first diagnosis to the date of death caused by glioma or the last date of follow-up, and cases of mortality unrelated to glioma were excluded from the present study.

Isolation of exosomes from serum. The extraction and purification of exosomes from blood serum and were performed as previously described (9). The serum was extracted from blood samples immediately. The collected serum was aliquoted and stored in liquid nitrogen. The samples were centrifuged to remove cells and other debris in the serum, first at $3,000 \mathrm{xg}$ for $30 \mathrm{~min}$ at room temperature, then at $10,000 \mathrm{x}$ for $20 \mathrm{~min}$ at room temperature. Subsequently, microvesicles that were larger than exosomes were removed from supernatants by centrifuging at $100,000 \times \mathrm{g}$ for $30 \mathrm{~min}$ at $4^{\circ} \mathrm{C}$. Subsequently, the supernatants were harvested, and again centrifuged at $10,000 \times \mathrm{g}$ for $70 \mathrm{~min}$ at $4^{\circ} \mathrm{C}$. Finally, the supernatants were gently decanted, and the exosome sediments were resuspended in PBS. The extracted exosomal samples were stored at $-80^{\circ} \mathrm{C}$ until further experiments.

Exosome characterization. The morphology and the size of the exosomes were imaged by Transmission Electron Microscopy (TEM). For TEM, collected exosomes were resuspended in $2 \%$ paraformaldehyde (Sigma-Aldrich; Merck KGaA) and spread onto carbon/Formvar-coated grid (Ted Pella, Inc.) for $30 \mathrm{~min}$ at room temperature. The grid was then washed with DPBS and fixed with $1 \%$ glutaraldehyde (Sigma-Aldrich; Merck KGaA). TEM images were captured with a JEOL JEM-2100F microscope (JEOL, Ltd). Magnification, x10,000.

miRNA isolation from exosomes and quantitative (q)PCR. The MirVana microRNA isolation kit (Thermo Fishes Scientific, Inc.) was used to isolate miRNAs from exosomes in accordance with the manufacturer's protocol. The isolated RNA was reverse transcribed and amplified using the TaqMan MicroRNA Reserve Transcription kit (Applied Biosystem; Thermo Fishes Scientific, Inc.). For the synthesis of cDNA, the reaction mixtures were incubated at $37.8^{\circ} \mathrm{C}$ for $60 \mathrm{~min}$ and $95^{\circ} \mathrm{C}$ for $5 \mathrm{~min}$, and then held at $4^{\circ} \mathrm{C}$. The cDNA specimens were stored at $-20^{\circ} \mathrm{C}$ until polymerase chain reaction (PCR). qPCR was performed using the QuantiTect SYBR-Green PCR mixture (Invitrogen; Thermo Fisher Scientific, Inc.) as described previously $(9,25)$. The reaction mixtures were incubated at $95^{\circ} \mathrm{C}$ for $10 \mathrm{~min}$, followed by 40 cycles of $95^{\circ} \mathrm{C}$ for $15 \mathrm{sec}, 56^{\circ} \mathrm{C}$ for $30 \mathrm{sec}$ and $72^{\circ} \mathrm{C}$ for $35 \mathrm{sec}$. miR-16, which is stably expressed across samples, was used as an endogenous control. The following primers were used: miR-210 forward, 5'-TTGACCTGTGCGTGTGACA-3' and reverse, 5'-TATGGT TGTTCTGCTCTCTGTCTC-3'; miR-16-1 forward, 5'-CGC CTGTAGCAGCACGTAA-3' and reverse, 5'-CAGAGCAGG GTCCGAGGTA-3'. The expression levels of the miRNAs were normalized to U6, and quantified using the $2^{-\Delta \Delta \mathrm{Cq}}$ method (25).

Immunohistochemistry and analysis. Immunohistochemical staining was used to evaluate the expression levels of HIF-1 $\alpha$ and CA9. Briefly, paraffin-embedded and formalin-fixed samples were cut into $4-\mu \mathrm{m}$ sections, which were then processed for immunohistochemistry. The sections were de-waxed, rehydrated, blocked with hydrogen peroxide, 
Table I. Association between clinical characteristics and the exo-miR-210 level.

\begin{tabular}{|c|c|c|c|}
\hline \multirow[b]{2}{*}{ Parameter } & \multirow[b]{2}{*}{ Patients (n) } & \multicolumn{2}{|c|}{ Exo-miR-210 } \\
\hline & & Mean expression & P-value \\
\hline Sex & & & 0.724 \\
\hline Male & 52 & 0.0030 & \\
\hline Female & 39 & 0.0034 & \\
\hline Age, years & & & 0.479 \\
\hline$\leq 45$ & 46 & 0.0029 & \\
\hline$>45$ & 45 & 0.0035 & \\
\hline Tumor size, cm & & & 0.539 \\
\hline$\leq 5$ & 28 & 0.0030 & \\
\hline$>5$ & 63 & 0.0032 & \\
\hline Relapse & & & $0.023^{\mathrm{a}}$ \\
\hline No & 42 & 0.0023 & \\
\hline Yes & 49 & 0.0040 & \\
\hline WHO grade & & & $0.001^{\mathrm{a}}$ \\
\hline I-II & 32 & 0.0010 & \\
\hline III-IV & 59 & 0.0044 & \\
\hline KPS score & & & 0.332 \\
\hline$\leq 90$ & 41 & 0.0033 & \\
\hline$>90$ & 50 & 0.0031 & \\
\hline
\end{tabular}

${ }^{a} \mathrm{P}<0.05$ (ANOVA). The median age was 45 years. WHO grade, World Health Organization grade (2); KPS, Karnofsky Performance Status (49); exo-miR-210, exosomal-microRNA-210; WHO, World Health Organization.

and the antigens were retrieved in a microwave in $10 \mathrm{mM}$ citrate buffer ( $\mathrm{pH} \mathrm{6.0)}$ for $10 \mathrm{~min}$ and cooled to room temperature. In order to block non-specific staining the tissue was incubated in blocking buffer, $1 \mathrm{ml}$ Protein blocking buffer (cat. no. ab126587; Abcam) diluted in $9 \mathrm{ml}$ PBS, for $30 \mathrm{~min}$ at room temperature. Following antigen retrieval for $20 \mathrm{~min}$ in citrate buffer $(0.01 \mathrm{M}$ citric acid, $\mathrm{pH}$ 6.0) at room temperature, sections were incubated overnight at $4^{\circ} \mathrm{C}$ with the primary antibody (1:100). HIF-1 $\alpha$ (cat. no. ab51608) and CA9 (cat. no. ab216021) antibodies were purchased from Abcam. Anti-mouse immunoglobulin G (IgG)-horseradish peroxidase (HRP) and anti-goat IgG-HRP goat anti-rabbit secondary antibodies (1:200; cat. no. ab205718; Santa Cruz Biotechnology, Inc.) was incubated with the sections for $1 \mathrm{~h}$ at room temperature, and 3,3'-diaminobenzidine buffer was used for signal detection. The sections were scored blindly by two pathologists using a light microscope at x200 magnification.

Either citrate buffer $\mathrm{pH} 6$ or EDTA buffer $\mathrm{pH} 9$ was used to perform antigen retrieval prior to cooling at $4{ }^{\circ} \mathrm{C}$ for 20 min. Subsequently, 3\% hydrogen peroxide was applied to block endogenous peroxidase activity for $10 \mathrm{~min}$. Primary antibodies (1:20 dilution) were incubated with samples for $15 \mathrm{~min}$ at room temperature. 3,3'-diaminobenzidine substrate was added for $5 \mathrm{~min}$ at room temperature until color developed prior to washing in running water for $10 \mathrm{~min}$. Slides were then counterstained in $10 \%$ hematoxylin at room temperature for $60 \mathrm{sec}$ and blued with Scotts' tap water before being dehydrated through a series of graded alcohols. Cover slips were applied using distrene, plasticizer and xylene. H-score was used to determine the expression of HIF- $1 \alpha$ and CA9: A combination of the intensity (0-3) and percentage of cells stained, with a range of 0-300. HIF-1 $\alpha$ scores only included nuclear expression and CA9 scores contained the staining of the nucleus and cytoplasm, as previously described (26).

Statistical analysis. SPSS version 13.0 (SPSS, Inc.) was used to assess and analyze statistical data. All quantitative data are presented as the mean \pm standard deviation (SD). Univariate and multivariate analysis of variance were performed. ANOVA was performed to analyze the significance between two groups (such as low-grade vs. high-grade glioma), and Student-Newman-Keuls test was performed to calculate the P-values. Kaplan-Meier analysis, a log-rank test and Cox regression analysis were used to calculate survival rates and predict factors associated with survival. $\mathrm{P}<0.05$ was considered to indicate a statistically significant difference. Technical and biological triplicates of each experiment were performed.

\section{Results}

Clinical characteristics of patients. Exosomal samples from the serum were collected from 91 patients with glioma and 50 healthy volunteers as a control. Patients with glioma included 12 patients with WHO grade I pilocytic astrocytoma, 20 patients with WHO grade II diffuse astrocytoma, 22 patients with WHO grade III anaplastic astrocytoma and 37 patients with WHO grade IV primary GBM. A total of 58 patients received radio-chemotherapy after surgery. At the last follow-up, 72 patients had succumbed to primary glioma, including 3 patients in the WHO grade I, 14 patients in WHO grade II, 20 patients in WHO grade III and 35 patients in WHO grade IV groups. The 1- and 2-year overall survival (OS) rates were 91.2 and $57.1 \%$, respectively.

Exo-miR-210 in the serum may serve as a diagnostic biomarker in glioma. A previous study demonstrated that serum miR-210 was overexpressed in patients with glioma, and high levels of serum miR-210 have been identified to be associated with worse overall survival (27). The present study aimed to further explore whether serum exo-miR-210 may serve as a diagnostic or prognostic biomarker for patients with glioma. Data from exosome characterization are shown in Fig. S1. Initially, levels of serum exo-miR-210 were analyzed by qPCR to compare the differences between patients with glioma $(n=91)$ and healthy controls $(n=50)$. Following normalization to miR-16, there was a significant difference in the expression levels of serum exo-miR-210 between patients with glioma and healthy controls $(\mathrm{P}<0.01$; Fig. 1A). Notably, patients with low-grade (I-II) glioma exhibited relatively low levels of serum exo-miR-210 compared with patients with high-grade (III-IV) glioma $(\mathrm{P}<0.01$; Fig. 1B). Collectively, the data suggested that serum exo-miR-210 was abnormally overexpressed in the serum of patients with glioma and increased with increasing grades of glioma; therefore, it maybe used as a biomarker for the diagnosis of glioma. 

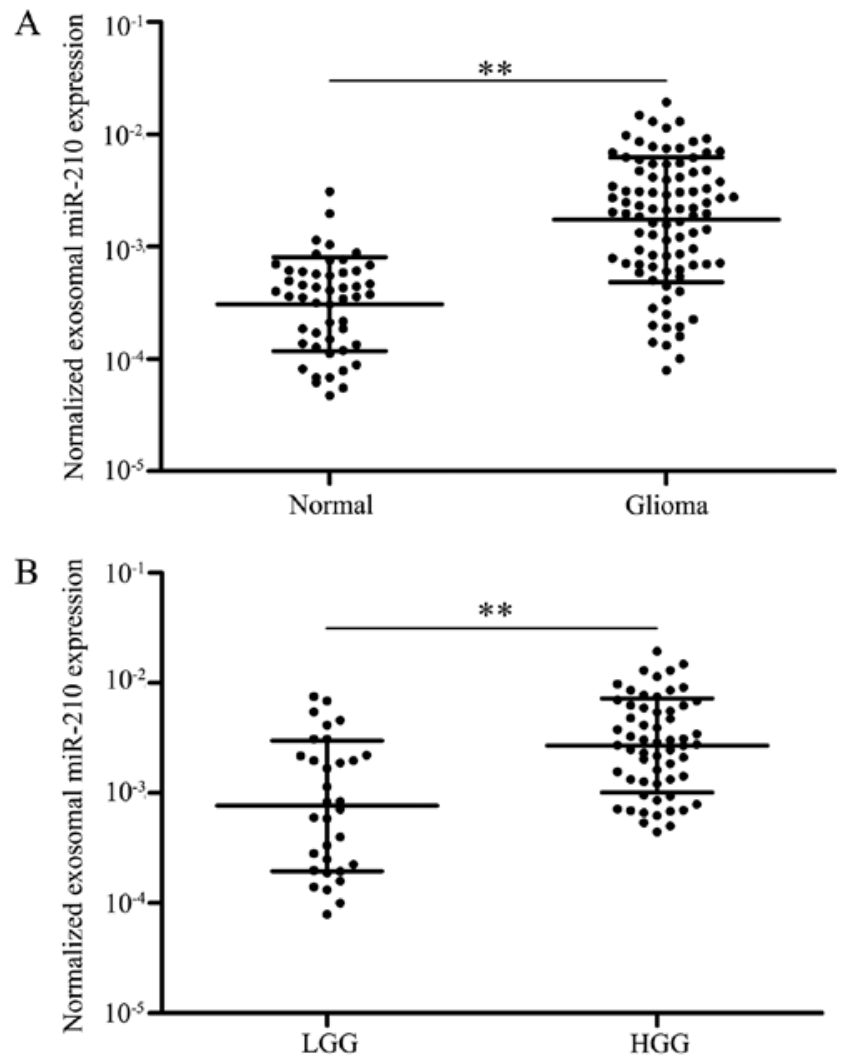

Figure 1. Expression of exo-miR-210 in serum samples. (A) Exo-miR-210 expression in patients with glioma and healthy controls analyzed by reverse transcription-quantitative PCR. (B) Serum exo-miR-210 expression in LGG and HGG. ${ }^{* *} \mathrm{P}<0.01$. exo-, exosomal-; HGG, high-grade glioma; LGG, low-grade glioma; miR-210, microRNA-210.

Expression levels of exo-miR-210 in the serum are associated with specific clinicopathological features. The putative associations between expression levels of exo-miR-210 and different clinicopathological features were explored. All patients were separated into two groups (low and high exo-miR-210 expression) according to the median concentration of exo-miR-210. The percentages of patients with glioma included in the high expression group of exo-miR-210 increased with grade, with 16.7, 20.0, 59.1 and $73.0 \%$ of patients with grade I, II, II and IV, respectively, being included in the high expression group. There was no statistically significant association identified between the level of serum exo-miR-210 and other clinicopathological parameters, including sex, KPS and age at diagnosis $(\mathrm{P}>0.05$; Table I). Advanced pathological grades were statistically significantly associated with serum exo-miR-210 expression $(\mathrm{P}<0.01$; Table I).

Serum exo-miR-210 levels reflect the dynamics of GBM. Subsequently, 20 patients with GBM from the original group were selected, and the expression levels of serum exo-miR-210 were detected pre- and post-operation. The gap period between the pre-and post-operation period was fixed at 1-2 weeks. Notably, serum exo-miR-210 levels were markedly decreased following curative surgical resection (Fig. 2A). Furthermore, the expression levels of serum exo-miR-210 were compared after surgery and at tumor recurrence in 10 patients with recurring GBM. Although relatively low levels of exo-miR-210 were
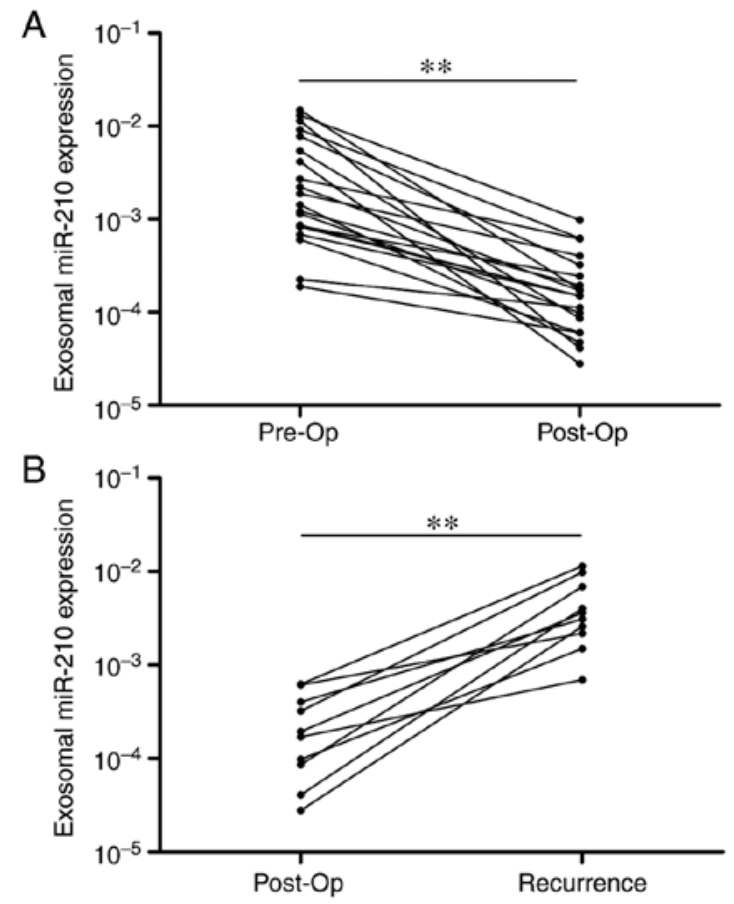

Figure 2. Serum exo-miR-210 levels reflect tumor dynamics. (A) Serum exo-miR-210 expression levels before and after surgery. miR-210 levels were significantly lower in the post-op samples than in the pre-op samples. (B) Comparison of serum exo-miR-210 levels in samples obtained post-op and after recurrence. A significant increase in serum exo-miR-210 levels was identified at recurrence. ${ }^{* *} \mathrm{P}<0.01$. exo-, exosomal-; miR-210, microRNA-210; post-op, postoperative; pre-op, preoperative.

found at the post-operation time point, a significant increase was detected at the time of GBM recurrence (Fig. 2B). Overall, serum exo-miR-210 secreted and released by GBM cancer cells could reflect tumor dynamics during treatment.

Expression of serum exo-miR-210 as a potential diagnostic and prognostic biomarker for glioma. To evaluate the diagnostic value of exo-miR-210 expression in patients with glioma, the present study applied receiver operating characteristic curve analysis and calculated the corresponding area under the curve (AUC) values to verify the potential diagnosis and discriminatory accuracy of exo-miR-210. As shown in Fig. 3, serum exo-miR-210 levels were robust in distinguishing patients with glioma from healthy controls, with an AUC value of 0.856 (95\% CI, 0.795-0.917), whereas the sensitivity, specificity, and positive and negative predictive values to discriminate patients with glioma were $83.2,94.3,93.5$ and $70.3 \%$, respectively.

Our previous study demonstrated that exo-miR-210 expression is associated with glioma grades, which suggests that exo-miR-210 may be an important predictor of poor prognosis in higher-grade compared with lower-grade gliomas (27). In this cohort, patients with high levels of exo-miR-210 exhibited a poor survival rate in contrast to patients with low expression levels of exo-miR-210 according to Kaplan-Meier survival analysis (Fig. 4). Subsequently, Cox regression analyses were carried out to assess the prognostic value of serum exo-miR-210 levels for OS. The results showed a significant association between serum exo-miR-210 levels and OS in patients with glioma $(\mathrm{P}<0.01$; hazard ratio, 3.63 ; 95\% CI, 


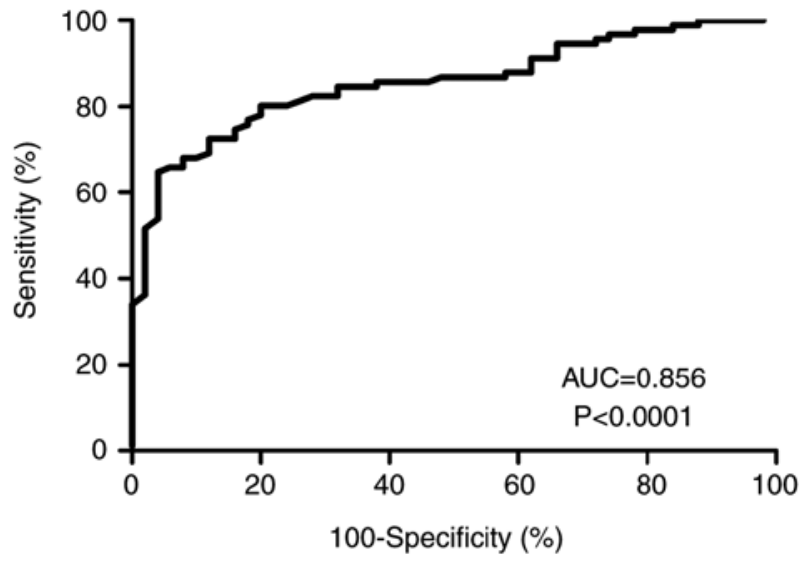

Figure 3. Receiver operating characteristic curve analysis based on serum exosomal microRNA-210 levels to distinguish patients with glioma from normal controls. AUC, area under the curve.

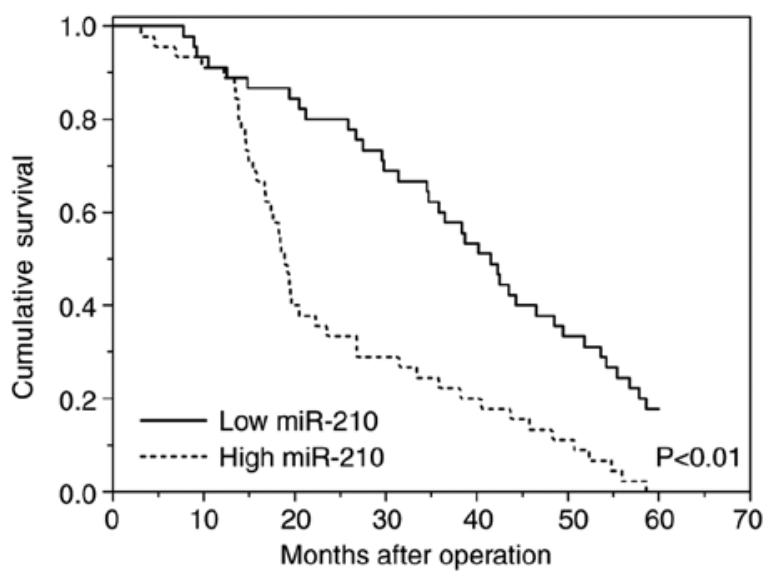

Figure 4. Kaplan-Meier survival curves for patients with glioma and high or low serum expression levels of exosomal miR-210. miR-210, microRNA-210.

1.54-5.67; Table II). Statistically significant associations were also observed in pathological grades. No significant association was observed in any of the other clinicopathological parameters tested, including sex, KPS and age at diagnosis (Table II). Overall, levels of serum exo-miR-210 secreted by glioma cells could be used as excellent indexes to differentiate patients with glioma from non-glioma patients, and as a valuable and novel biomarker in the diagnosis and prognosis of human glioma.

Expression levels of serum exo-miR-210 are associated with hypoxia. A number of studies $(28,29)$ have demonstrated that the overexpression of miR-210 is regulated in a HIF-1 $\alpha$-dependent manner in numerous types of cancer. Integrated analysis of microRNA, mRNA and chromatin immunoprecipitation-sequencing data has revealed that miR-210 expression under hypoxia is regulated at the transcriptional and post-transcriptional levels, with the presence of HIF-1 $\alpha$ binding sites (30). Previous studies have demonstrated that miR-210 is associated with tumor hypoxia and predicts benefit from hypoxic modification $(31,32)$. Based on these previous studies, the present study examined the association between serum exo-miR-210 expression and HIF-1 $\alpha$ and CA9 levels. Representative images of immunohistochemistry of HIF-1 $\alpha$ and CA9 are shown in Fig. 5A. When exo-miR-210 expression was compared with conventional hypoxic markers, higher serum exo-miR-210 expression was accompanied by high HIF-1 $\alpha$ and CA9 protein levels (Fig. 5B). Based on the results of the present study, it can be concluded that serum exo-miR-210 could be used as a potential biomarker of hypoxia.

\section{Discussion}

Biomarkers could provide a more accurate assessment to predict the diagnosis and prognosis of various types of cancer according to tumor biology. Exosomes are a reservoir of valuable and novel biomarkers for cancer diagnosis and prognosis. Exosomes secreted to the extracellular microenvironment are involved in the cell-cell communication to neighboring and distant cells, favoring secretion of growth factors, cytokines and angiopoietic factors, which can induct tumor proliferation, metastasis, therapeutic efficacy and immune responses (33). Exosomes can be isolated from blood or other bodily fluids to reveal progression of cancer occurring in the body (34). miRNA can be horizontally transferred into the extracellular microenvironment by exosomes to modify the microenvironment. Exosomal miRNAs are emerging as an identified group of messengers and effectors in intercellular communication (35).

Serum exo-miRNAs can function as novel non-invasive biomarkers for the detection and monitoring of various types of cancer and putatively as therapeutic targets. Levels of serum exo-miR-21 are increased and associated with the progression and aggressiveness of esophageal squamous cell cancer (36), whereas levels of serum exo-miR-19a have been identified to serve as prognostic biomarkers for recurrence in patients with colorectal cancer (37). Exo-miR-21 extracted from cerebrospinal fluids is associated with a poor prognosis and tumor recurrence in patients with glioma (38). Levels of serum exo-miR-1290 and exo-miR-375 are associated with castration resistance in prostate cancer (39). miR-223-3p encapsulated by exosomes has been identified as an important biomarker for the early detection of invasive breast cancer (40). Exo-miR-423-5p has been reported as a minimally invasive biomarker in the diagnosis of gastric cancer, and can promote tumor growth and metastasis by targeting SUFU negative regulator of hedgehog signaling (41). The present study obtained data, which suggested that the exo-miR-210 level in serum was a diagnostic, prognostic and predictive biomarker for glioma, and associated with hypoxic conditions. miR-210, which is a regulator of several cellular functions, dependent on or independent of hypoxia signaling, is involved in numerous biological processes of numerous tumors throughout the human body (42). miR-210 has been identified as an activator of tumor initiation and development, and its high levels are associated with a negative clinical outcome (43). It has been reported to be involved in cell survival, differentiation, angiogenesis, metabolism and cell cycle control $(15,44)$. In addition, elevated miR-210 levels increase the proliferation and decrease apoptosis of GBM cells by targeting polypyrimidine tract binding protein 3 (45). miR-210 extracted from the peripheral blood is a promising and minimally invasive biomarker that can be employed to diagnose and predict the outcome of glioma (27). 
Table II. Univariate and multivariate analyses of prognostic parameters in patients with glioma.

\begin{tabular}{|c|c|c|c|c|}
\hline \multirow[b]{2}{*}{ Parameter } & \multicolumn{2}{|c|}{ Univariate analysis } & \multicolumn{2}{|c|}{ Multivariate analysis } \\
\hline & P-value & $\mathrm{HR}(95 \% \mathrm{CI})$ & P-value & $\operatorname{HR}(95 \% \mathrm{CI})$ \\
\hline Age ( $\leq 45$ vs. $>45)$ & 0.77 & $1.26(0.64-1.91)$ & 0.48 & $1.03(0.51-1.67)$ \\
\hline Sex (male vs. female) & 0.64 & $1.09(0.45-1.56)$ & 0.59 & $1.13(0.57-1.78)$ \\
\hline KPS ( $\leq 90$ vs. $>90)$ & 0.19 & $1.37(0.84-1.98)$ & 0.43 & $0.98(0.60-1.37)$ \\
\hline WHO grade (I-II vs. III-IV) & $<0.01$ & $7.88(3.54-12.53)$ & $<0.01$ & $11.25(5.34-19.67)$ \\
\hline Exosomal miR-210 & $<0.01$ & $3.63(1.54-5.67)$ & $<0.01$ & $4.31(1.94-8.13)$ \\
\hline
\end{tabular}

The median age was 45 years. HR, hazard ratio; KPS, Karnofsky Performance Status (49); WHO grade, World Health Organization tumor grade (2); miR-210, microRNA-210; WHO, World Health Organization.

A

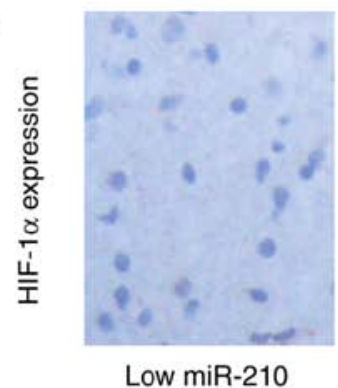

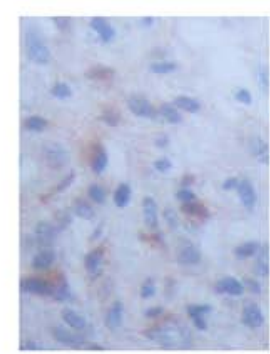

High miR-210

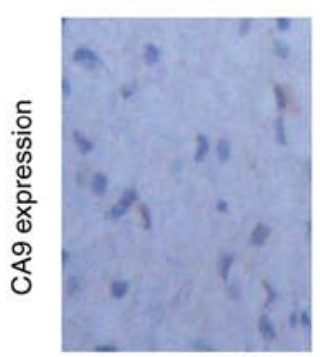

Low miR-210

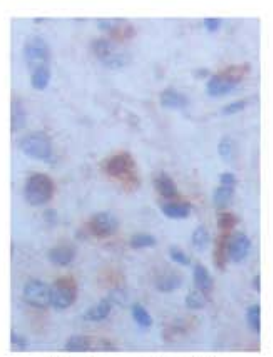

High miR-210
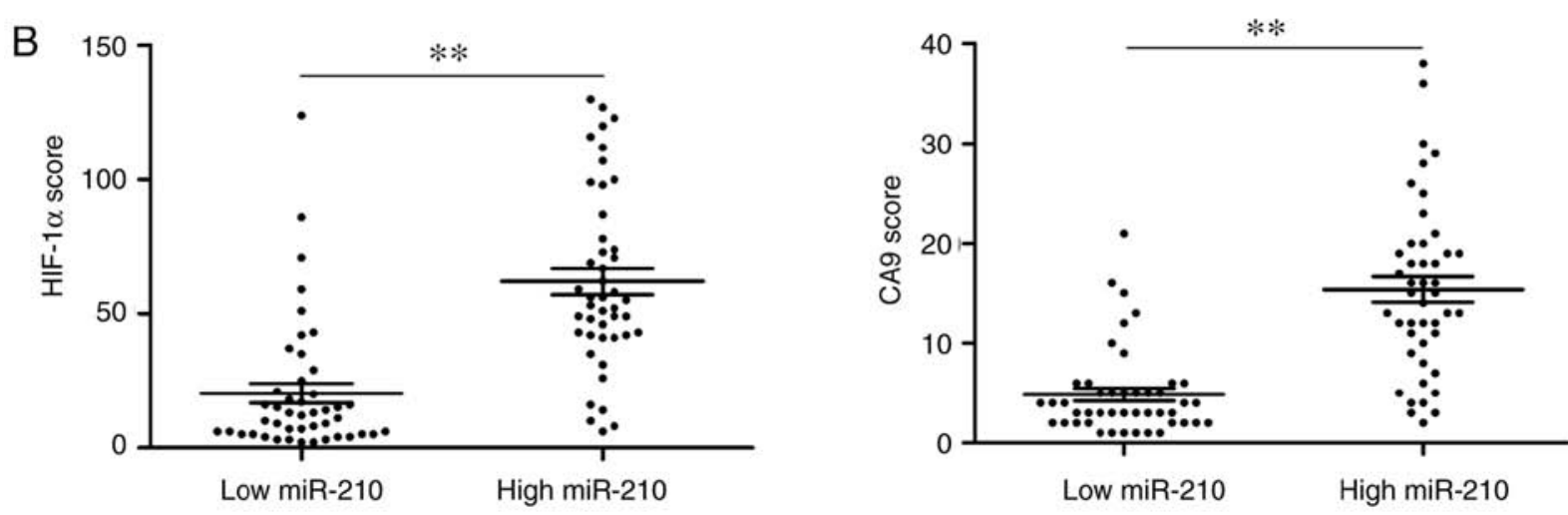

Figure 5. High serum levels of exosomal miR-210 are strongly associated with markers of tumor hypoxia. (A) HIF-1 $\alpha$ and (B) CA9. ${ }^{* *} \mathrm{P}<0.01$. CA9, carbonic anhydrase 9; HIF-1 $\alpha$, hypoxia-inducible factor $1 \alpha$; miR-210, microRNA-210.

miR-210, which has been reported as one of the abnormally upregulated miRNAs in hypoxic cells, is highly conserved and required for cell survival (46). In addition, since other studies have reported that the level of miR-210 is dependent on HIF-1 $\alpha$ regulation, increased expression of miR-210 in tissues and serum has become a signature for tumor hypoxia $(47,48)$.

The aim of the present study was to examine whether exo-miR-210 is a potential non-invasive biomarker for the diagnosis and prognosis of glioma and associated with hypoxic conditions. Firstly, a significant difference was identified in the expression levels of serum exo-miR-210 between patients with glioma and healthy controls. Importantly, serum exo-miR-210 exhibited a relatively low level in low-grade (I-II) compared with high grade (III-IV) glioma. Additionally, serum exo-miR-210 levels were markedly decreased following curative surgical resection and significantly increased at the time of GBM recurrence. Notably, high levels of exo-miR-210 were associated with a poor survival rate compared with low expression levels of exo-miR-210 according to Kaplan-Meier survival analysis. Subsequently, Cox regression analyses revealed that serum exo-miR-210 levels were associated with the OS of patients with glioma. Finally, overexpression of exo-miR-210 was significantly associated with high protein levels of HIF-1a and CA9 and reflected hypoxia in patients with glioma. Although the present study discussed the potential use of exo-miR-210 as a biomarker in glioma, the underlying mechanism of exo-miR-210 in hypoxic conditions has not been identified. Future studies are needed to explore the unique properties of exo-miR-210 and allow the development of highly sensitive strategies to rapidly and non-invasively monitor the pathological condition of patients with glioma. 
In summary, serum exo-miR-210 expression is abnormally elevated in glioma, particularly GBM, and may function as a novel biomarker for predicting diagnosis and progression of patients with glioma. Further larger independent studies are required prior to applying the results of the present study to clinical practice.

\section{Acknowledgements}

Not applicable.

\section{Funding}

The present study was supported by the China National Natural Scientific Fund (grant nos. 81772690, 81502654 and 81872468).

\section{Availability of data and materials}

The datasets used and/or analyzed during the present study are available from the corresponding author on reasonable request.

\section{Authors' contributions}

TX conceived and designed the study. FL and XY performed the experiments.

\section{Ethics approval and consent to participate}

The present study was approved by the Medical School of Chinese PLA (Beijing, China) and Tianjin Huanhu Hospital (Tianjin, China). All participants provided written informed consent prior to recruitment.

\section{Patient consent for publication}

Not applicable.

\section{Competing interests}

The authors declare that they have no competing interests.

\section{References}

1. Savage N: Searching for the roots of brain cancer. Nature 561: S50-S51, 2018

2. Louis DN, Perry A, Reifenberger G, von Deimling A, Figarella-Branger D, Cavenee WK, Ohgaki H, Wiestler OD, Kleihues P and Ellison DW: The 2016 world health organization classification of tumors of the central nervous system: A summary. Acta Neuropathol 131: 803-820, 2016.

3. Ahmed SU, Carruthers R, Gilmour L, Yildirim S, Watts C and Chalmers AJ: Selective inhibition of parallel DNA damage response pathways optimizes radiosensitization of glioblastoma stem-like cells. Cancer Res 75: 4416-4428, 2015.

4. Colwell N, Larion M, Giles AJ, Seldomridge AN, Sizdahkhani S, Gilbert MR and Park DM: Hypoxia in the glioblastoma microenvironment: Shaping the phenotype of cancer stem-like cells Neuro Oncol 19: 887-896, 2017.

5. Majmundar AJ, Wong WJ and Simon MC: Hypoxia-inducible factors and the response to hypoxic stress. Mol Cell 40: 294-309, 2010.

6. Wu D, Potluri N, Lu J, Kim Y and Rastinejad F: Structural integration in hypoxia-inducible factors. Nature 524: 303-308, 2015 .
7. Semenza GL: Hypoxia-inducible factors: Mediators of cancer progression and targets for cancer therapy. Trends Pharmacol Sci 33: 207-214, 2012.

8. Sadri N and Zhang PJ: Hypoxia-inducible factors: Mediators of cancer progression; Prognostic and therapeutic targets in soft tissue sarcomas. Cancers (Basel) 5: 320-333, 2013.

9. Lan F, Qing Q, Pan Q, Hu M, Yu H and Yue X: Serum exosomal miR-301a as a potential diagnostic and prognostic biomarker for human glioma. Cell Oncol (Dordr) 41: 25-33, 2018.

10. Yue X, Cao D, Lan F, Pan Q, Xia T and Yu H: MiR-301a is activated by the Wnt/ $\beta$-catenin pathway and promotes glioma cell invasion by suppressing SEPT7. Neuro Oncol 18: 1288-1296, 2016.

11. Lan F, Yu H, Hu M, Xia T and Yue X: miR-144-3p exerts anti-tumor effects in glioblastoma by targeting c-Met. J Neurochem 135: 274-286, 2015.

12. Xu K, Zhan Y, Yuan Z, Qiu Y, Wang H, Fan G, Wang J, Li W, Cao Y, Shen X, et al: Hypoxia induces drug resistance in colorectal cancer through the HIF-1 $\alpha / \mathrm{miR}-338-5 \mathrm{p} / \mathrm{IL}-6$ feedback loop. Mol Ther 2019 (Epub ahead of print).

13. Zhou Y, Xu Q, Shang J, Lu L and Chen G: Crocin inhibits the migration, invasion, and epithelial-mesenchymal transition of gastric cancer cells via miR-320/KLF5/HIF-1 $\alpha$ signaling. J Cell Physiol 234: 17876-17885, 2019.

14. Zhao Q, Li Y, Tan BB, Fan LQ, Yang PG and Tian Y: HIF-1 $\alpha$ induces multidrug resistance in gastric cancer cells by inducing MiR-27a. PLoS One 10: e0132746, 2015.

15. Ke HL, Li WM, Lin HH, Hsu WC, Hsu YL, Chang LL, Huang CN, Li CC, Chang HP, Yeh HC, et al: Hypoxia-regulated MicroRNA-210 overexpression is associated with tumor development and progression in upper tract urothelial carcinoma. Int J Med Sci 14: 578-584, 2017.

16. He C, Wang L, Zhang J and $\mathrm{Xu} \mathrm{H}$ : Hypoxia-inducible microRNA-224 promotes the cell growth, migration and invasion by directly targeting RASSF8 in gastric cancer. Mol Cancer 16: $35,2017$.

17. Ge X, Liu X, Lin F, Li P, Liu K, Geng R, Dai C, Lin Y, Tang W, Wu Z, et al: MicroRNA-421 regulated by HIF-1 $\alpha$ promotes metastasis, inhibits apoptosis, and induces cisplatin resistance by targeting E-cadherin and caspase-3 in gastric cancer. Oncotarget 7: 24466-24482, 2016.

18. Benej M, Pastorekova S and Pastorek J: Carbonic anhydrase IX: Regulation and role in cancer. Subcell Biochem 75: 199-219, 2014.

19. Ziffels B, Stringhini M, Probst P, Fugmann T, Sturm T and Neri D: Antibody-based delivery of cytokine payloads to carbonic anhydrase IX leads to cancer cures in immunocompetent tumor-bearing mice. Mol Cancer Ther 18: 1544-1554, 2019.

20. Meng W, Hao Y, He C, Li L and Zhu G: Exosome-orchestrated hypoxic tumor microenvironment. Mol Cancer 18: 57, 2019.

21. Hoshino A, Costa-Silva B, Shen TL, Rodrigues G, Hashimoto A, Tesic Mark M, Molina H, Kohsaka S, Di Giannatale A, Ceder S, et al: Tumour exosome integrins determine organotropic metastasis. Nature 527: 329-335, 2015.

22. Nouraee N, Khazaei S, Vasei M, Razavipour SF, Sadeghizadeh M and Mowla SJ: MicroRNAs contribution in tumor microenvironment of esophageal cancer. Cancer Biomarkers 16: 367-376, 2016.

23. Xiao GY, Cheng CC, Chiang YS, Cheng WT, Liu IH and Wu SC: Exosomal miR-10a derived from amniotic fluid stem cells preserves ovarian follicles after chemotherapy. Sci Rep 6: 23120, 2016.

24. Khalid MA, Achakzai IK, Ahmed Khan S, Majid Z, Hanif FM, Iqbal J, Laeeq SM and Luck NH: The use of karnofsky performance status (KPS) as a predictor of 3 month post discharge mortality in cirrhotic patients. Gastroenterol Hepatol Bed Bench 11: 301-305, 2018.

25. Yue X, Lan F, Hu M, Pan Q, Wang Q and Wang J: Downregulation of serum microRNA-205 as a potential diagnostic and prognostic biomarker for human glioma. J Neurosurg 124: 122-128, 2016.

26. Eustace A, Irlam JJ, Taylor J, Denley H, Agrawal S, Choudhury A, Ryder D, Ord JJ, Harris AL, Rojas AM, et al: Necrosis predicts benefit from hypoxia-modifying therapy in patients with high risk bladder cancer enrolled in a phase III randomised trial. Radiother Oncol 108: 40-47, 2013.

27. Lai NS, Wu DG, Fang XG, Lin YC, Chen SS, Li ZB and Xu SS: Serum microRNA-210 as a potential noninvasive biomarker for the diagnosis and prognosis of glioma. Br J Cancer 112: 1241-1246, 2015

28. Dang K and Myers KA: The role of hypoxia-induced miR-210 in cancer progression. Int J Mol Sci 16: 6353-6372, 2015. 
29. Yang W, Ma J, Zhou W, Zhou X, Cao B, Fan D and Hong L: Biological implications and clinical value of mir-210 in gastrointestinal cancer. Expert Rev Gastroenterol Hepatol 11: 539-548, 2017.

30. Camps C, Saini HK, Mole DR, Choudhry H, Reczko M, Guerra-Assunção JA, Tian YM, Buffa FM, Harris AL, Hatzigeorgiou AG, et al: Integrated analysis of microRNA and mRNA expression and association with HIF binding reveals the complexity of microRNA expression regulation under hypoxia. Mol Cancer 13: 28, 2014.

31. Agrawal R, Pandey P, Jha P, Dwivedi V, Sarkar C and Kulshreshtha R: Hypoxic signature of microRNAs in glioblastoma: Insights from small RNA deep sequencing. BMC Genomics 15: 686, 2014.

32. Irlam-Jones JJ, Eustace A, Denley H, Choudhury A, Harris AL, Hoskin PJ and West CM: Expression of miR-210 in relation to other measures of hypoxia and prediction of benefit from hypoxia modification in patients with bladder cancer. Br J Cancer 115 571-578, 2016.

33. Li D, Liu J, Guo B, Liang C, Dang L, Lu C, He X, Cheung HY, $\mathrm{Xu} \mathrm{L}, \mathrm{Lu} \mathrm{C}$, et al: Osteoclast-derived exosomal miR-214-3p inhibits osteoblastic bone formation. Nat Commun 7: 10872, 2016.

34. Munagala R, Aqil F and Gupta RC: Exosomal miRNAs as biomarkers of recurrent lung cancer. Tumour Biol 37 : 10703-10714, 2016.

35. Zhou W, Fong MY, Min Y, Somlo G, Liu L, Palomares MR, Yu Y Chow A, O'Connor ST, Chin AR, et al: Cancer-secreted miR-105 destroys vascular endothelial barriers to promote metastasis. Cancer Cell 25: 501-515, 2014

36. Tanaka Y, Kamohara H, Kinoshita K, Kurashige J, Ishimoto T, Iwatsuki M, Watanabe $M$ and Baba $\mathrm{H}$ : Clinical impact of serum exosomal microRNA-21 as a clinical biomarker in human esophageal squamous cell carcinoma. Cancer 119: 1159-1167, 2013.

37. Matsumura T, Sugimachi K, Iinuma H, Takahashi Y, Kurashige J, Sawada G, Ueda M, Uchi R, Ueo H, Takano Y, et al: Exosomal microRNA in serum is a novel biomarker of recurrence in human colorectal cancer. Br J Cancer 113: 275-281, 2015.

38. Shi R, Wang PY, Li XY, Chen JX, Li Y, Zhang XZ, Zhang CG, Jiang T, Li WB, Ding W and Cheng SJ: Exosomal levels of miRNA-21 from cerebrospinal fluids associated with poor prognosis and tumor recurrence of glioma patients. Oncotarget 6 : 26971-26981, 2015.
39. Huang X, Yuan T, Liang M, Du M, Xia S, Dittmar R, Wang D, See W, Costello BA, Quevedo F, et al: Exosomal miR-1290 and miR-375 as prognostic markers in castration-resistant prostate cancer. Eur Urol 67: 33-41, 2015.

40. Yoshikawa M,IinumaH,Umemoto Y, Yanagisawa T, Matsumoto A and Jinno H: Exosome-encapsulated microRNA-223-3p as a minimally invasive biomarker for the early detection of invasive breast cancer. Oncol Lett 15: 9584-9592, 2018.

41. Yang H, Fu H, Wang B, Zhang X, Mao J, Li X, Wang M, Sun Z, Qian $\mathrm{H}$ and $\mathrm{Xu}$ W: Exosomal miR-423-5p targets SUFU to promote cancer growth and metastasis and serves as a novel marker for gastric cancer. Mol Carcinog 57: 1223-1236, 2018.

42. Bavelloni A, Ramazzotti G, Poli A, Piazzi M, Focaccia E, Blalock W and Faenza I: MiRNA-210: A current overview. Anticancer Res 37: 6511-6521, 2017.

43. Ren CX, Leng RX, Fan YG, Pan HF, Wu CH and Ye DQ: MicroRNA-210 and its theranostic potential. Expert Opin Ther Targets 20: 1325-1338, 2016.

44. Bonora M, Wieckowsk MR, Chinopoulos C, Kepp O, Kroemer G, Galluzzi L and Pinton P: Molecular mechanisms of cell death: Central implication of ATP synthase in mitochondrial permeability transition. Oncogene 34: 1608, 2015.

45. Zhang S, Lai N, Liao K, Sun J and Lin Y: MicroRNA-210 regulates cell proliferation and apoptosis by targeting regulator of differentiation 1 in glioblastoma cells. Folia Neuropathol 53: 236-244, 2015.

46. Kulshreshtha R, Ferracin M, Wojcik SE, Garzon R, Alder H, Agosto-Perez FJ, Davuluri R, Liu CG, Croce CM, Negrini M, et al: A microRNA signature of hypoxia. Mol Cell Biol 27: 1859-1867, 2007.

47. Ying Q, Liang L, Guo W, Zha R, Tian Q, Huang S, Yao J, Ding J, Bao M, Ge C, et al: Hypoxia-inducible microRNA-210 augments the metastatic potential of tumor cells by targeting vacuole membrane protein 1 in hepatocellular carcinoma. Hepatology 54: 2064-2075, 2011

48. Cheng HH, Mitchell PS, KrohEM, Dowell AE, Chéry L, Siddiqui J, Nelson PS, Vessella RL, Knudsen BS, Chinnaiyan AM, et al: Circulating microRNA profiling identifies a subset of metastatic prostate cancer patients with evidence of cancer-associated hypoxia. PLoS One 8: e69239, 2013

49. Martin RC, Gerstenecker A, Nabors LB, Marson DC and Triebel KL: Impairment of medical decisional capacity in relation to karnofsky performance status in adults with malignant brain tumor. Neurooncol Pract 2: 13-19, 2015. 